Revista Ciências Exatas e Naturais, Vol.20, nº.1, Jan/Jun, 2018

\title{
Propriedades trigonométricas em triângulos Pitagóricos
}

\section{Trigonometric properties on Pythagorean triangles}

\author{
Maurício de Araujo Ferreira \\ Universidade Estadual de Feira de Santana - UEFS, Feira de Santana, BA \\ maferreira@uefs.br \\ Calebe Miranda da Silva \\ Universidade Estadual de Feira de Santana - UEFS, Feira de Santana, BA \\ calfeiranova@hotmail.com
}

Resumo: O objetivo central deste artigo é mostrar que não existe um triângulo retângulo com lados e ângulos (em graus) inteiros. O célebre Teorema de Pitágoras estabelece que os lados de um triângulo retângulo satisfazem a equação $a^{2}+b^{2}=c^{2}$. Uma terna de números inteiros $(a, b, c)$ satisfazendo essa equação é chamada de Tripla Pitagórica. Propriedades trigonométricas dos ângulos agudos desses triângulos motivam a definição de ângulos pitagóricos, que são ângulos tais que o seno e o cosseno são racionais. É mostrado que os únicos ângulos pitagóricos com medida inteira em graus são múltimos de $90^{\circ}$. A demonstração dada aqui é elementar e utiliza apenas relações trigonométricas e divisibilidade, podendo ser apresentada para um estudante de nível médio. Por fim, mostra-se que o conjunto dos ângulos pitagóricos é denso na reta real.

Palavras-chave: Triplas pitagóricas; ângulos pitagóricos; trigonometria.

Abstract: The aim of this paper is to prove that there is no right-angled triangle with integers sides and integers angles (in degrees). The famous Pythagorean Theorem states that the sides of a right triangle satisfy the equation $a^{2}+b^{2}=c^{2}$. A triple of integers $(a, b, c)$ satisfying this equation is called a Pythagorean Triple. Trigonometric properties of the acute angles of these triangles motivate the definition of Pythagorean angles, which are angles that the sine and the cosine are rational. It is shown that the only Pythagorean angles with integer measure in degrees are multiples of 90 . The demonstration given here is elementary and uses only trigonometric relations and divisibility, and can be presented to a middle-level student. Finally, it is shown that the set of Pythagorean angles is dense in the real line.

Key words: Pythagorean Theorem; pythagorean angles; trigonometry.

\section{Introdução}

Conforme Boyer [1], os babilônios, no período entre 1900 e 1600 a.E.C., já conheciam o fato de que em um triângulo retângulo, a soma do quadrado das medidas dos catetos é igual ao quadrado da medida da hipotenusa. A tabuleta de argila Plimpton 322 (ver [2]), que foi escrita neste período, possuem sequências de números inteiros satisfazendo essa propriedade. Entretanto, a tradição é unânime em atribuir a Pitágoras a descoberta 
independente do teorema sobre triângulos retângulos hoje universalmente conhecidos pelo seu nome: Teorema de Pitágoras. Acredita-se que a primeira demonstração geral do teorema pode ter sido dada por Pitágoras. As ternas de números inteiros positivos $(a, b, c)$ que satisfazem a equação

$$
a^{2}+b^{2}=c^{2}
$$

são denominadas triplas ou ternas pitagóricas. Estas correspondem aos comprimentos dos lados de um triângulo retângulo de lados inteiros pelo Teorema de Pitágoras, como ilustrado na Figura 1. Exemplos de ternas pitagóricas são $(3,4,5)$ e $(8,15,17)$. Triplas pitagóricas satisfazem propriedades interessantes: se $(a, b, c)$ é uma tripla pitagórica, então $a, b$ ou $c$ é sempre multiplo de 5 e $a b$ é multiplo de 6 . Para demonstrações, ver [3].

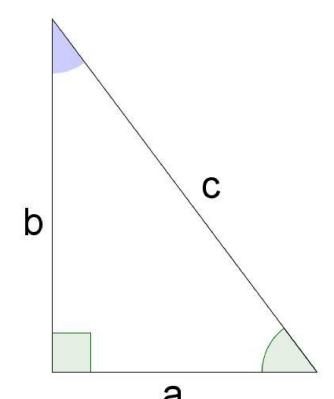

Figura 1. Triângulo retângulo.

Nota-se que se $(a, b, c)$ é uma tripla pitagórica, então $(k a, k b, k c)$ também é uma tripla pitagórica para todo inteiro $k$. Neste caso, os triâgulos retângulos associados $(a, b, c) \mathrm{e}$ $(k a, k b, k c)$ são semelhantes, isto é, tem os mesmos ângulos internos. Também, se existir um primo $p$ tal que $p \mid \operatorname{mdc}(a, b)$, tem-se que $p \mid\left(a^{2}+b^{2}\right)=c^{2}$, logo $p \mid c$. Assim, $\left(\frac{a}{p}, \frac{b}{p}, \frac{c}{p}\right)$ também é uma tripla pitagórica. Chega-se à mesma conclusão se $p \mid \operatorname{mdc}(a, c)$ ou $p \mid \operatorname{mdc}(b, c)$. Este fato motiva a definição de tripla pitagórica primitiva, que é uma tripla pitagórica $(a, b, c)$ cujos termos são dois a dois primos entre si. Note que $a$ e $b$ tem paridades distintas e $c$ é sempre ímpar.

O Teorema 1.1 abaixo fornece uma fórmula geral para ternas pitagóricas primitivas. Para demonstração ver [3].

Teorema 1.1 A terna $(a, b, c)$ é uma terna pitagórica primitiva com a par se, e somente se, são da forma

$$
a=2 m n, \quad b=m^{2}-n^{2} e \quad c=m^{2}+n^{2} .
$$

com $m d c(m, n)=1$ e $m$ e $n$ de paridades distintas.

Segue do Teorema 1.1 que existem infinitas ternas pitagóricas primitivas, isto é, existem infinitos triângulos retângulos com lados inteiros que não são semelhantes entre si. Na seção 2 serão estudadas propriedades dos ângulos internos desses triângulos, e na seção 3, mostrase um resultado mais geral sobre a infinitude das triplas pitagóricas primitivas, a saber, o conjunto dos ângulos pitagóricos é denso em $\mathbb{R}$. 
Revista Ciências Exatas e Naturais, Vol.20, nº.1, Jan/Jun, 2018

\section{2 Ângulos pitagóricos}

Um triângulo retângulo com lados inteiros positivos $a, b$ e $c$ é dito um triângulo pitagórico. Considere o triângulo pitagórico como na Figura 2. Note que sen $\alpha=\frac{a}{c}$ e $\cos \alpha=\frac{b}{c}$. Assim, ambos $\cos \alpha$ e sen $\alpha$ são números racionais. Essa propriedade motiva a definição de ângulo pitagórico. Um número real $\alpha$ é dito um ângulo pitagórico se sen $\alpha$ e $\cos \alpha$ são números racionais.

Note que $\alpha=k \frac{\pi}{2}$ é um ângulo pitagórico para todo inteiro $k$, pois sen $\alpha$ e $\cos \alpha$ assume valores $-1,0$ ou 1 .

Se $(a, b, c)$ é uma terna pitagórica, então os ângulos agudos internos do triângulo retângulo de lados a,b e c, como na Figura 2, é pitagórico. De fato, sen $\alpha=\cos \beta=\frac{a}{c}$ e $\cos \alpha=$ $\operatorname{sen} \beta=\frac{b}{c}$. Em particular, os ângulos internos do triângulo pitagórico de lados 3,4 e 5 são ângulos pitagóricos.

Tem-se que $\frac{\pi}{6}, \frac{\pi}{4}$ e $\frac{\pi}{3}$ não são ângulos pitagóricos pois $\cos \frac{\pi}{6}=\operatorname{sen} \frac{\pi}{3}=\frac{\sqrt{3}}{2}$ e $\cos \frac{\pi}{4}=\frac{\sqrt{2}}{2}$, que são números irracionais.

Observa-se que se um ângulo agudo $\alpha$ é pitagórico, tem-se que $\alpha$ é um dos ângulos internos de um triângulo pitagórico. De fato, se $\operatorname{sen} \alpha=\frac{a}{b}$ e $\cos \alpha=\frac{c}{d}$, com $a, b, c$ e $d$ inteiros, então, da relação $\operatorname{sen}^{2} \alpha+\cos ^{2} \alpha=1$, tem se $\left(\frac{a}{b}\right)^{2}+\left(\frac{c}{d}\right)^{2}=1$. Segue que $(a d)^{2}+(b c)^{2}=(b d)^{2}$. Como pode-se escrever sen $\alpha=\frac{a d}{b d}$ e $\cos \alpha=\frac{b c}{b d}$, conclui-se que $\alpha$ é um dos ângulos internos do triângulo de catetos $a d$ e $b c$ e hipotenusa $b d$.

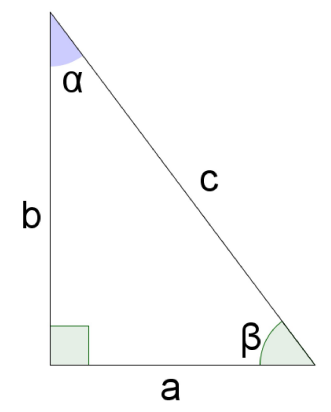

Figura 2. Triângulo retângulo e ângulos pitagóricos agudos.

Proposição 2.1 Seja $\alpha \in \mathbb{R}$. Então:

(i) $\alpha$ é um ângulo pitagórico se, e somente se, o seu complementar $\frac{\pi}{2}-\alpha$ é ângulo pitagórico.

(ii) $\alpha$ é um ângulo pitagórico se, e somente se, $k \pi \pm \alpha$ é ângulo pitagórico. Em particular, $\alpha$ é um ângulo pitagórico se, e somente se, o seu suplementar é ângulo pitagórico.

(i) Seja $\beta=\frac{\pi}{2}-\alpha$. Note que $\operatorname{sen} \alpha=\cos \beta$ e $\cos \alpha=\operatorname{sen} \beta$ (ver Figura 3). Logo $\alpha$ é ângulo pitagórico se, e somente se, $\beta$ é ângulo pitagórico.

(ii) Seja $\theta=k \pi \pm \alpha$. Tem-se que $\alpha$ e $\theta$ tem os mesmos senos e cossenos a menos de sinal. Assim, $\alpha$ é ângulo pitagórico se, e somente se, $\theta$ é ângulo pitagórico. Por fim, note que o suplementar de $\alpha$ é $\pi-\alpha$. 


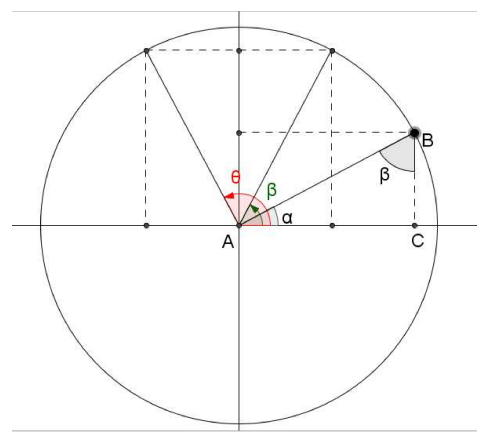

Figura 3. Ângulo complementar e ângulo suplementar.

Proposição 2.2 Se $\alpha$ e $\beta$ são ângulos pitagóricos, então $\alpha+\beta$ e $\alpha-\beta$ são ângulos pitagóricos.

Suponha que $\alpha$ e $\beta$ são ângulos pitagóricos. Então pode-se escrever sen $\alpha=\frac{a_{1}}{c_{1}}, \cos \alpha=\frac{b_{1}}{c_{1}}$, $\operatorname{sen} \beta=\frac{a_{2}}{c_{2}}$ e $\cos \beta=\frac{b_{2}}{c_{2}}$, com $a_{1}, a_{2}, b_{1}, b_{2}, c_{1}, c_{2}$ inteiros. Assim tem-se

$$
\begin{aligned}
\operatorname{sen}(\alpha+\beta) & =\operatorname{sen} \alpha \cos \beta+\cos \alpha \operatorname{sen} \beta \\
& =\frac{a_{1}}{c_{1}} \frac{b_{2}}{c_{2}}+\frac{b_{1}}{c_{1}} \frac{a_{2}}{c_{2}} \\
& =\frac{a_{1} b_{2}+b_{1} a_{2}}{c_{1} c_{2}}
\end{aligned}
$$

Da mesma forma, tem-se

$$
\begin{aligned}
\cos (\alpha+\beta) & =\cos \alpha \cos \beta-\operatorname{sen} \alpha \operatorname{sen} \beta \\
& =\frac{b_{1}}{c_{1}} \frac{b_{2}}{c_{2}}-\frac{a_{1}}{c_{1}} \frac{a_{2}}{c_{2}} \\
& =\frac{b_{1} b_{2}-a_{1} a_{2}}{c_{1} c_{2}} .
\end{aligned}
$$

Logo $\operatorname{sen}(\alpha+\beta)$ e $\cos (\alpha+\beta)$ são racionais, portanto $\alpha+\beta$ é um ângulo pitagórico. Da mesma forma,

$$
\begin{aligned}
\operatorname{sen}(\alpha-\beta) & =\operatorname{sen}, \alpha \cos (-\beta)+\cos \alpha \operatorname{sen}(-\beta) \\
& =\operatorname{sen} \alpha \cos \beta-\cos \alpha \operatorname{sen} \beta \\
& =\frac{a_{1}}{c_{1}} \frac{b_{2}}{c_{2}}-\frac{b_{1}}{c_{1}} \frac{a_{2}}{c_{2}} \\
& =\frac{a_{1} b_{2}-b_{1} a_{2}}{c_{1} c_{2}} .
\end{aligned}
$$


Revista Ciências Exatas e Naturais, Vol.20, nº.1, Jan/Jun, 2018

Também,

$$
\begin{aligned}
\cos (\alpha-\beta) & =\cos \alpha \cos (-\beta)-\operatorname{sen} \alpha \operatorname{sen}(-\beta) \\
& =\cos \alpha \cos \beta+\operatorname{sen} \alpha \operatorname{sen} \beta \\
& =\frac{b_{1}}{c_{1}} \frac{b_{2}}{c_{2}}+\frac{a_{1}}{c_{1}} \frac{a_{2}}{c_{2}} \\
& =\frac{b_{1} b_{2}+a_{1} a_{2}}{c_{1} c_{2}} .
\end{aligned}
$$

Logo $\operatorname{sen}(\alpha-\beta)$ e $\cos (\alpha-\beta)$ são racionais, portanto $\alpha-\beta$ é um ângulo pitagórico.

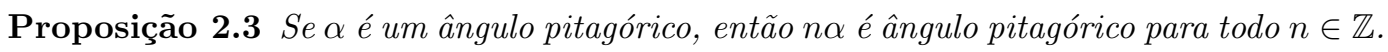

O caso $n \geq 0$ é feito por indução em $n$. Para $n=0$ ou $n=1$ não há nada o que fazer. Suponhamos que a proposição é válida para $n=k$, isto é, $k \alpha$ é pitagórico. Como, $(k+1) \alpha=$ $k \alpha+\alpha$, segue da Proposição 2.2 que $(k+1) \alpha$ é pitagórico. Portanto, $n \alpha$ é ângulo pitagórico para todo inteiro positivo $n$.

Suponhamos agora $n<0$. Note que $\cos (n \alpha)=\cos (-n \alpha)$ e $\operatorname{sen}(n \alpha)=-\operatorname{sen}(-n \alpha)$. Como $-n>0$, tem-se pelo caso anterior que $\cos (-n \alpha)$ e $-\operatorname{sen}(-n \alpha)$ são números racionais, $\operatorname{logo} \cos (n \alpha)$ e sen $(n \alpha)$ também são racionais. Portanto, n $\alpha$ é ângulo pitagórico.

Os únicos ângulos pitagóricos $\alpha^{\circ} \operatorname{com} \alpha$ sendo um número inteiro são os múltiplos de $90^{\circ}$. Pelo Exemplo 2 os múltiplos de $90^{\circ}$ são ângulos pitagóricos. Deve-se mostrar que os ângulos que não são múltiplo de $90^{\circ}$ não são ângulos pitagóricos. Pela Proposição 3 , podemos supor sem perda de generalidade que $0 \leqslant \alpha \leqslant 45$, pois todos os demais ângulos podem ser escritos da forma $k \pi \pm \alpha$ ou $k \pi-\left(\frac{\pi}{2}-\alpha\right)$ com $k$ inteiro e $0 \leqslant \alpha \leqslant 45$.

Pelo Exemplo 2 tem se que $30^{\circ}, 45^{\circ}$ e $60^{\circ}$ não são pitagóricos. Assim pela Proposição 2.3 tem-se que os divisores inteiros de $30^{\circ}, 45^{\circ}$ e $60^{\circ}$ não são ângulos pitagóricos. Ou seja, os ângulos $1^{\circ}, 2^{\circ}, 3^{\circ}, 4^{\circ}, 5^{\circ}, 6^{\circ}, 9^{\circ}, 10^{\circ}, 12^{\circ}, 15^{\circ}, 20^{\circ}, 30^{\circ}$ e $45^{\circ}$ não são pitagóricos. Aplicando a Proposição 2.1, concluímos que $150^{\circ}, 135^{\circ}, 120^{\circ}, 210^{\circ}, 240^{\circ}$ e $330^{\circ}$ não são ângulos pitagóricos. Portanto seus divisores inteiros também não são pitagóricos. Assim, concluímos que $7^{\circ}, 8^{\circ}, 11^{\circ}, 14^{\circ}, 16^{\circ}, 21^{\circ}, 22^{\circ}, 24^{\circ}, 25^{\circ}, 27^{\circ}, 33^{\circ}, 35^{\circ}, 40^{\circ}$ e $42^{\circ}$ não são pitagóricos, pois $25^{\circ}$ é divisor de $150^{\circ} ; 27^{\circ}$ é divisor de $135^{\circ} ; 8^{\circ}$ e $24^{\circ}$ são divisores de $120^{\circ}$; $7^{\circ}, 14^{\circ}, 21^{\circ}, 35^{\circ}$ e $42^{\circ}$ são divisores de $210^{\circ} ; 11^{\circ}, 22^{\circ}$ e $33^{\circ}$ são divisores de $330^{\circ}$, e $16^{\circ}$ e $40^{\circ}$ são divisores de $240^{\circ}$.

Aplicando novamente a Proposição 2.1 com os ângulos obtidos no parágrafo anterior, tem-se que $88^{\circ}$ não é ângulo pitagórico, pois é complementar de $2^{\circ}$. Portanto podemos concluir que seu divisores não são ângulos pitagóricos, isto é, $44^{\circ}$ também não é ângulo pitagóricos por ser divisor 88. Com o mesmo argumento vamos indicar quais ângulos $\alpha$ não são pitagóricos com $0^{\circ}<\alpha<45^{\circ}$, observe a Tabela 1 .

Para concluir a demonstração, pela Tabela 1 resta verificar que os ângulos $18^{\circ}$ e $36^{\circ}$ não são ângulos pitagóricos. Vamos calcular o seno e cosseno dos ângulos de $18^{\circ}$ e $36^{\circ}$. Para este cálculo usaremos como referência [4]. A demonstração será refeita aqui para tornar o trabalho tão auto suficiente quanto possível.

Considere o triângulo isósceles $A B C$ como da Figura $4 \operatorname{com} \overline{A B}=\overline{A C}=1$ e $B \widehat{A} C=36^{\circ}$. Traçando a bissetriz $C D$ de $A \widehat{C} B$, podemos calcular todos os ângulos da figura. Como os triângulos $C D B$ e $C D A$ são isósceles, temos $\overline{B C}=\overline{C D}=\overline{D A}$ e, como os triângulos $C D B$ e $A B C$ são semelhantes, temos

$$
\frac{\overline{C B}}{\overline{D B}}=\frac{\overline{C A}}{\overline{C B}}
$$


Tabela 1. Ângulos não pitagóricos

\begin{tabular}{cccc}
\hline$\alpha$ & $\begin{array}{c}\text { Complementar } \\
\text { de } \alpha\end{array}$ & $\begin{array}{c}\text { Divisores do } \\
\text { complementar de } \alpha\end{array}$ & $\begin{array}{c}\text { Novos ângulos } \\
\text { não pitagóricos }\end{array}$ \\
\hline $2^{\mathrm{o}}$ & $88^{\circ}$ & $1,2,4,8,11,22,44$ e 88 & $44^{\circ}$ \\
$3^{\circ}$ & $87^{\circ}$ & $1,3,29$ e 87 & $29^{\circ}$ \\
$4^{\circ}$ & $86^{\circ}$ & $1,2,43$ e 86 & $43^{\circ}$ \\
$5^{\circ}$ & $85^{\circ}$ & $1,5,17$ e 65 & $17^{\circ}$ \\
$6^{\circ}$ & $84^{\circ}$ & $1,2,3,4,6,7,12,14,21,28,42$ e 84 & $28^{\circ}$ \\
$8^{\circ}$ & $82^{\circ}$ & $1,2,41$ e 82 & $41^{\circ}$ \\
$12^{\circ}$ & $78^{\circ}$ & $1,2,3,6,13,26,39$ e 78 & $13^{\circ}, 26^{\circ}$ e $39^{\circ}$ \\
$14^{\circ}$ & $76^{\circ}$ & $1,2,4,19,38$ e 76 & $19^{\circ}$ e $38^{\circ}$ \\
$16^{\circ}$ & $74^{\circ}$ & $1,2,37$ e 74 & $37^{\circ}$ \\
$21^{\circ}$ & $69^{\circ}$ & $1,3,23$ e 69 & $23^{\circ}$ \\
$22^{\circ}$ & $68^{\circ}$ & $1,24,17,34$ e 68 & $34^{\circ}$ \\
$26^{\circ}$ & $64^{\circ}$ & $1,2,4,16,32$ e 64 & $32^{\circ}$ \\
$28^{\circ}$ & $62^{\circ}$ & $1,2,31$ e 62 & $31^{\circ}$ \\
\hline
\end{tabular}

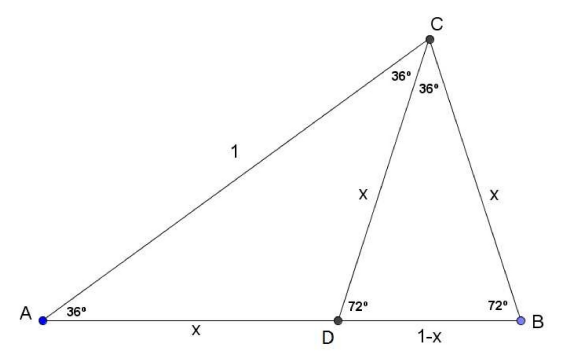

Figura 4. Triângulo isósceles

isto é,

$$
\frac{x}{1-x}=\frac{1}{x}
$$

o que nos leva a equação do quadrática $x^{2}+x-1=0$, cuja as soluções são $x_{1}=\frac{\sqrt{5}-1}{2} \mathrm{e}$ $x_{2}=\frac{-\sqrt{5}-1}{2}$. Logo $x=\frac{\sqrt{5}-1}{2}$, por ser a única solução positiva.

Traçando a altura $A H$ do triângulo isóscele $A B C$ (Figura 5), temos sen $18^{\circ}=\frac{\overline{H B}}{A B}=\frac{x}{2}$, ou seja,

$$
\operatorname{sen} 18^{\circ}=\frac{\sqrt{5}-1}{4}
$$

Pela relação fundamental $\operatorname{sen}^{2} 18^{\circ}+\cos ^{2} 18^{\circ}=1$, concluímos que

$$
\cos 18^{\circ}=\frac{\sqrt{10+2 \sqrt{5}}}{4} .
$$

Portanto o ângulo $18^{\circ}$ não é pitagórico. 
Revista Ciências Exatas e Naturais, Vol.20, nº.1, Jan/Jun, 2018

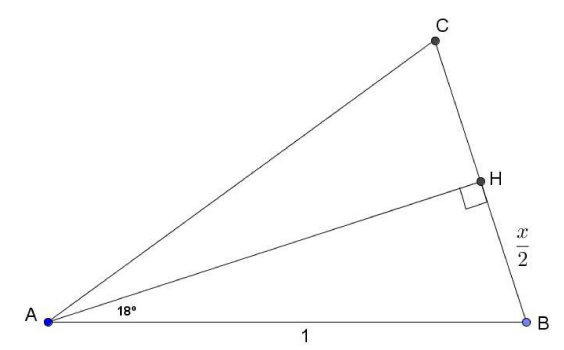

Figura 5. Altura $A H$ do triângulo isósceles $A B C$.

Podemos agora calcular o seno de $36^{\circ}$ pela formula $\operatorname{sen} 2 \theta=2 \operatorname{sen} \theta \cos \theta$. De fato,

$$
\begin{aligned}
\operatorname{sen} 36^{\circ} & =\frac{2(\sqrt{5}-1)}{4} \frac{\sqrt{10+2 \sqrt{5}}}{4} \\
& =\frac{\sqrt{10-2 \sqrt{5}}}{4} .
\end{aligned}
$$

Pela relação fundamental $\operatorname{sen}^{2} 36^{\circ}+\cos ^{2} 36^{\circ}=1$, obtemos

$$
\cos 36^{\circ}=\frac{\sqrt{6+2 \sqrt{5}}}{4} .
$$

Portanto o ângulo $36^{\circ}$ também não é pitagórico.

\section{Densidade dos ângulos pitagóricos em $\mathbb{R}$}

Já vimos que o conjunto dos ângulos pitagóricos é infinito. Nesta seção vamos obter um resultado mais geral, a saber, mostraremos que entre quaisquer dois números reais existe sempre um ângulo pitagórico.

Um subconjunto $A \subseteq \mathbb{R}$ é dito denso, se para todo intervalo $(a, b) \subseteq \mathbb{R}$ existe $c \in A$ tal que $c \in(a, b)$. Exemplos de conjuntos densos em $\mathbb{R}$ são o conjunto dos racionais $\mathbb{Q}$ e o conjunto dos irracionais $\mathbb{R} \backslash \mathbb{Q}(\operatorname{ver}[5$, Teorema 4$])$.

$\mathrm{O}$ conjunto dos ângulos pitagóricos é denso em $\mathbb{R}$.

Sejam $a, b \in \mathbb{R}$ com $a<b$. Por simplificação, podemos supor que $a, b \in(0, \pi / 2)$. O resultado geral segue por simetria no ciclo trigonométrico. Considere a função

$$
f:\left(0, \frac{\pi}{2}\right) \rightarrow \mathbb{R}, \text { definida por } f(x)=\frac{\operatorname{sen} x+1}{\cos x} .
$$

Note que $f$ é crescente pois $f(x)=\operatorname{tg} x+\sec x$ e ambas as funções tangente e secante são crescentes no intervalo $\left(0, \frac{\pi}{2}\right)$. Logo, $f$ é injetiva. O conjunto imagem da função $f$ é $\operatorname{Im}(f)=(0,+\infty)$. Assim, $f$ tem inversa crescente que é dada por

$$
g:(0,+\infty) \rightarrow\left(0, \frac{\pi}{2}\right), \text { definida por } g(x)=\operatorname{arctg}\left(\frac{x^{2}-1}{2 x}\right),
$$


pois

$$
\begin{aligned}
g \circ f(x) & =\operatorname{arctg}\left(\frac{\left(\frac{\operatorname{sen} x+1}{\cos x}\right)^{2}-1}{2\left(\frac{\operatorname{sen} x+1}{\cos x}\right)}\right) \\
& =\operatorname{arctg}\left(\frac{\frac{\operatorname{sen}^{2} x+2 \operatorname{sen} x+1-\cos ^{2} x}{\cos x}}{\frac{2 \operatorname{sen} x+2}{\cos x}}\right) \\
& =\operatorname{arctg}\left(\frac{\operatorname{sen}^{2} x+2 \operatorname{sen} x+\operatorname{sen}^{2} x}{(2 \operatorname{sen} x+2) \cos x}\right) \\
& =\operatorname{arctg}\left(\frac{\operatorname{sen} x(2 \operatorname{sen} x+2)}{\cos x(2 \operatorname{sen} x+2)}\right) \\
& =\operatorname{arctg}(\operatorname{tg} x) \\
& =x .
\end{aligned}
$$

Para o cálculo de $f \circ g(x)$, note que para $x \in\left(0, \frac{\pi}{2}\right)$, tem-se que

$$
f(x)=\operatorname{tg} x+\sec x=\operatorname{tg} x+\sqrt{1+\operatorname{tg}^{2} x} .
$$

Assim,

$$
\begin{aligned}
f \circ g(x) & =\frac{x^{2}-1}{2 x}+\sqrt{1+\left(\frac{x^{2}-1}{2 x}\right)^{2}} \\
& =\frac{x^{2}-1}{2 x}+\sqrt{\frac{4 x^{2}+x^{4}-2 x^{2}+1}{4 x^{2}}} \\
& =\frac{x^{2}-1}{2 x}+\frac{x^{2}+1}{2 x} \\
& =x .
\end{aligned}
$$

Como $f$ é crescente, tem-se que $f(a)<f(b)$. Como $\mathbb{Q}$ é denso em $\mathbb{R}$ então existe $c \in \mathbb{Q}$ tal que $f(a)<c<f(b)$. Aplicando $g$, que também é crescente, obtém-se $a<g(c)<b$. Resta verificar que $g(c)$ é um ângulo pitagórico. Mas $\operatorname{tg}(g(c))=\frac{c^{2}-1}{2 c}$. Então $\operatorname{sen}(g(c))=\frac{c^{2}-1}{c^{2}+1}$ e $\cos (g(c))=\frac{2 c}{c^{2}+1}$. Como $c$ é racional, conclui-se que $\operatorname{sen}(g(c))$ e $\cos (g(c))$ também são racionais. Portanto, $g(c)$ é ângulo pitagórico.

\section{Conclusão}

Conclui-se a partir do Teorema 2 que não existe um triângulo retângulo com lados e ângulos (em graus) inteiros. Por outro lado, obtém-se do Teorema 3 que existem infinitos ângulos pitagóricos e mais ainda, estes formam um conjunto denso na reta real. Isto significa que dado qualquer intervalo aberto $(a, b) \subset(0, \pi / 2)$ existe um triângulo pitagórico tal que um dos seus ângulos agudos (ângulo pitagórico) pertence ao intervalo $(a, b)$. Mais ainda, a função $g$ no supracitado teorema fornece uma fórmula para obtenção de ângulos pitagóricos. Entretanto, verifica-se que não é conhecida uma apresentação simples para os ângulos pitagóricos, como por exemplo os ângulos inteiros (quando medidos em graus), conforme visto no Teorema 2. Seria interessante se obter uma descrição completa dos ângulos pitagóricos. 
Revista Ciências Exatas e Naturais, Vol.20 , nº.1, Jan/Jun, 2018

\section{Referências}

[1] BOYER, C. B., História da matemática. Trad. Elza F. G. Sao Paulo: Edgard Blucher, 1974.

[2] Columbia University Libraries. History of Science, Mathematics, Technology, \# 158. Disponível em: $<$ http://www.columbia.edu/cu/lweb/eresources/exhibitions/treasures/html/158.html> Acesso em: 10 de novembro de 2017.

[3] MOREIRA, C. G. T. A., Tópicos de teoria dos números, Rio de Janeiro: SBM, 2012 .

[4] CARMO, M.P.; Morgado, A. C.; Wagner, E., Trigonometria/ Números Complexos - 3 ed. Rio de Janeiro: SBM, 2005.

[5] LIMA, E. L. Curso de Análise. 10. ed. Rio de Janeiro, RJ: Sociedade Brasileira de Matemática, 2002. Vol. 1. 